HEAD AND NECK

\title{
Long-term outcomes of alternating chemoradiotherapy in patients with advanced nasopharyngeal cancer: a single-centre experience over the last decade
}

\author{
Risultati a lungo termine di chemioradioterapia alternata in pazienti con carcinoma \\ rinofaringeo in stadio avanzato: esperienza di un singolo centro nell'ultima decade \\ S. SAIJOH ${ }^{1}$, T. MATSUZUKA ${ }^{1}$, H. SATO ${ }^{2}$, M. SUZUKI ${ }^{1}$, M. IKEDA ${ }^{1}$, R. SUZUKI ${ }^{1}$, Y. NAKAEGAWA ${ }^{1}$, K. OMORI ${ }^{1}$ \\ ${ }^{1}$ Department of Otolaryngology; ${ }^{2}$ Department of Radiation Oncology, Fukushima Medical University, Fukushima, Japan
}

\section{SUMMARY}

We assessed the long-term outcomes of alternating chemoradiotherapy (ACRT) using 5-fluorouracil and cisplatin (FP) in 25 patients with stage II or advanced nasopharyngeal cancer treated at our institution between April 1999 and April 2010. Median follow-up duration was 87 months (range 2-189). According to the 2009 TNM classification (UICC), six patients were in stage II, nine in stage III, and 10 in stage IV. Treatment completion, response and five-year survival rates were retrospectively assessed. ACRT was performed with a first course of chemotherapy administered followed by the initial round of radiotherapy (36 Gy). Then, a second course of chemotherapy with additional radiotherapy (20-30 Gy) was administered, followed by a final third course of chemotherapy. For chemotherapy, 5-fluorouracil (5-FU, $800 \mathrm{mg} / \mathrm{m}^{2} / 24 \mathrm{~h}$ ) was intravenously administered for five days, and cisplatin (CDDP, $50 \mathrm{mg} / \mathrm{m}^{2} / 24 \mathrm{~h}$ ) was administered on the last two days. Treatment completion rate was $96 \%$ (24 of 25 cases), and the response rate was 100\% (CR: 24 cases and PR: 1 case). Additionally, the five-year overall survival rate was $89.3 \%$. We have demonstrated that ACRT is an effective regimen to treat nasopharyngeal cancer, revealing higher treatment completion, response, and five-year overall survival rates compared with other combinatorial radiotherapy and chemotherapy treatment regimens.

KEY WORDS: Alternating chemoradiotherapy $\bullet$ Nasopharyngeal cancer $\bullet$ Cisplatin $\bullet 5$-fluorouracil

\section{RIASSUNTO}

Abbiamo valutato gli outcome a lungo termine di chemioradioterapia alternata (ACRT) utilizzando 5-fluorouracile e cisplatino (FP) in 25 pazienti con carcinoma in stadio II o avanzato del rinofaringe trattati nel nostro istituto tra l'Aprile del 1999 e l'A prile del 2010 . La durata media del follow up è stata di 87 mesi (range 2-189). In accordo con il TNM del 2009 (UICC), sei pazienti erano in stadio II, nove in stadio III e dieci in stadio IV. Sono stati valutati retrospettivamente il completamento del trattamento, la risposta ed i tassi di sopravvivenza a 5 anni. ACRT è stata eseguita utilizzando un primo ciclo di chemioterapia seguito da radioterapia (36 Gy), quindi un secondo ciclo di chemioterapia seguito da radioterapia (20-30 Gy), quindi un terzo ciclo di chemioterapia. Per la chemioterapia è stato utilizzato 5-florouracile endovenoso (5-FU, $800 \mathrm{mg} / \mathrm{m} 2 / 24 \mathrm{~h}$ ) per cinque giorni e cisplatino (CDDP, $\left.50 \mathrm{mg} / \mathrm{m}^{2} / 24 \mathrm{~h}\right)$ negli ultimi 2 giorni. Il tasso di completamento del trattamento è stato del 96\% (24 casi su 25), quello di risposta del 100\% (CR 24 casi, PR 1 caso). La sopravvivenza globale è stata dell' $89,3 \%$. Abbiamo dimostrato che ACRT è un regime efficace per il trattamento del carcinoma del rinofaringe, con un miglior tasso di completamento del trattamento, di risposta e di sopravvivenza a 5 anni se confrontato con altri protocolli di chemioradioterapia.

PAROLE CHIAVE: Chemioradioterapia alternata $\bullet$ Carcinoma del rinofaringe $\bullet$ 5-fluorouracile

Acta Otorhinolaryngol Ital 2018;38:103-108

\section{Introduction}

Almost all nasopharyngeal cancers are poorly differentiated squamous cell carcinomas or undifferentiated carcinoma and are more sensitive to radiotherapy compared with other head and neck cancers. Surgical treatment of nasopharyngeal cancer is difficult, and thus, various combination therapies of chemotherapy and radiotherapy are more viable management options. Specifically, adjuvant 
or neo-adjuvant chemotherapy with radiotherapy, concurrent chemoradiotherapy and alternating chemoradiotherapy have been shown to improve survival outcomes of nasopharyngeal cancer patients ${ }^{1-7}$. Since April 1999, our hospital has performed alternating chemoradiotherapy based on Fuwa's regimen ${ }^{1}$ for stage II or higher nasopharyngeal cancer patients. In this study, we highlight the efficacy of alternating chemoradiotherapy in these patients focusing on the following outcomes: (1) treatment completion rates, (2) response rates, and (3) five-year survival rates.

\section{Materials and methods}

\section{Patient criteria}

This study was approved by the Institutional Review Board and Ethics Committee of the Fukushima Medical University (Fukushima, Japan). Patients with untreated nasopharyngeal cancer who met the following criteria were enrolled in this study: (1) diagnosed with squamous cell carcinoma, or undifferentiated carcinoma; (2) exhibited stage II or advanced nasopharyngeal cancer; (3) free of any organ abnormalities (renal function: creatinine clearance $>60 \mathrm{~mL} / \mathrm{min}$ ); and (4) provided written, informed consent.

There were no patients excluded from criteria above between April 1999 and April 2010 at Fukushima Medical University Hospital.

\section{Patients}

We examined 25 patients histologically confirmed as having stage II, III, or IV type nasopharyngeal cancer.These patients underwent ACRT between April 1999 and April 2010 at Fukushima Medical University Hospital and were included in this retrospective analysis. According to the 2009 TNM staging classification system, cancer stages of the patients were as follows: stage IIA $(\mathrm{n}=2)$; stage IIB $(n=4)$; stage III $(n=9)$; stage IVA $(n=5)$; and stage IVB $(n=5)$. Patient characteristics are described in Table I. Nineteen patients were male, and six patients were female. The mean age was 46 years (range: $12-71$ years) at the time of diagnosis of the primary tumour. The median observation period was 87 months (range: 2-189 months). Progression-free survival and OS rates were analysed with the Kaplan-Meier method. The time to event was calculated from the beginning of the treatment.

\section{ACRT regimen}

During ACRT, the first course of chemotherapy with FP was performed before the initial round of radiotherapy.
Table I. Characteristics of nasopharyngeal cancer.

\begin{tabular}{|c|c|}
\hline Patient characteristics & Number of patients \\
\hline \multicolumn{2}{|l|}{ Sex } \\
\hline Male & 19 \\
\hline Female & 6 \\
\hline \multicolumn{2}{|l|}{ Performance status } \\
\hline 0 & 24 \\
\hline 1 & 1 \\
\hline 2 & 0 \\
\hline \multicolumn{2}{|l|}{ Stage } \\
\hline$\| \mathrm{A}$ & 2 \\
\hline \multicolumn{2}{|l|}{ T2aN0:2 } \\
\hline$\| \mathrm{B}$ & 4 \\
\hline \multicolumn{2}{|l|}{ T2aN1:2 T2bN1:2 } \\
\hline |l| & 9 \\
\hline \multicolumn{2}{|c|}{ T2aN2:1; T2bN2:5; T3N0:1; T3N1:1; T3N2:1 } \\
\hline IV A & 5 \\
\hline \multicolumn{2}{|l|}{ T4N0:1 T4N1:3 T4N2:1 } \\
\hline IV B & 5 \\
\hline \multicolumn{2}{|c|}{ T2bN3a:2; T2bN3b:1; T4N3a:2 } \\
\hline \multicolumn{2}{|c|}{ Histology } \\
\hline WHO type I & 3 \\
\hline WHO type II & 12 \\
\hline WHO type III & 10 \\
\hline \multicolumn{2}{|l|}{ Subsite } \\
\hline Postero-superior wall & 2 \\
\hline Lateral wall & 23 \\
\hline Inferior wall & 0 \\
\hline \multicolumn{2}{|l|}{ Follow up duration } \\
\hline 2-11 months & 1 \\
\hline $12-59$ months & 10 \\
\hline 60-190 months & 14 \\
\hline
\end{tabular}

One course of initial chemotherapy consisted of continuous intravenous administration of 5-fluorouracil (5-FU) at a dose of $800 \mathrm{mg} / \mathrm{m}^{2} / 24 \mathrm{~h}$ for five days (days 1-5) and cisplatin (CDDP) at a dose of $50 \mathrm{mg} / \mathrm{m}^{2} / 24 \mathrm{~h}$ for two days (days 6-7). The drip infusion of fluid replacement was established at $4 \mathrm{~L} /$ day during CDDP administration. A minimum urine volume of $2 \mathrm{~L}$ /day was maintained for five days after CDDP administration. Steroids, palonosetron and aprepitant were used as antiemetics against CDDP. The second and third course of chemotherapies were performed 2-3 days after completion of the initial and additional rounds of radiotherapy (described below). Our regimen is described in Figure 1. When serum creatinine levels were above $1.5 \mathrm{mg} / \mathrm{dL}$ on the scheduled date of chemotherapy, chemotherapy was not performed. In 


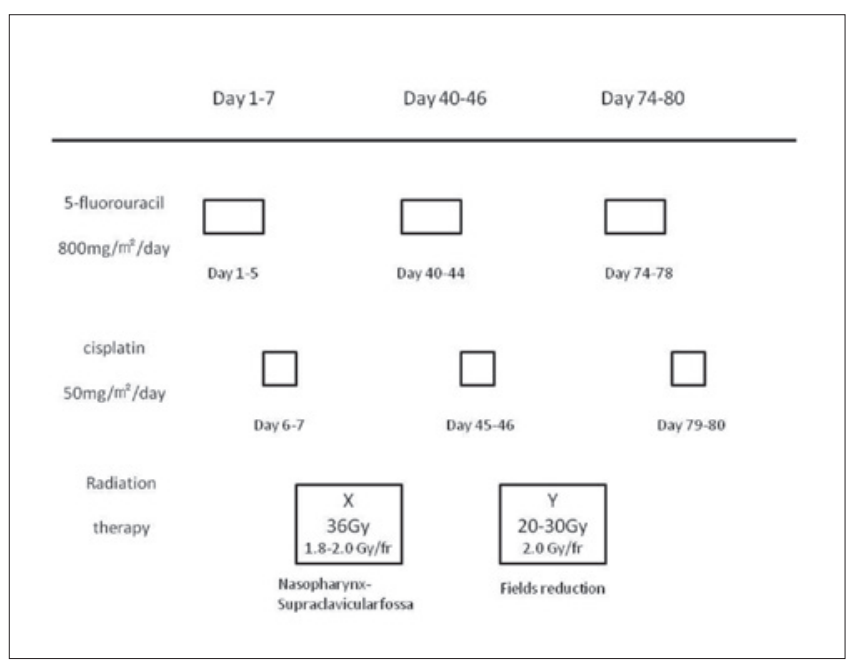

Fig. 1. Alternating chemoradiotherapy protocol. 5-FU, 5-fluorouracil, 800 $\mathrm{mg} / \mathrm{m} 2 /$ day on days 1-5; CDDP, cisplatin, $50 \mathrm{mg} / \mathrm{m} 2 /$ day on days 6-7; Field $X$, large field (from the Nasopharynx to the supraclavicular fossa), 1.8-2.0 Gy, 5f/w, total tumour dose, 36Gy; Field Y, fields reduction, 2.0Gy, 5f/w, total tumour dose, 20-30Gy.

addition, when a WBC count below $3,000 / \mathrm{mm}^{2}$ or a platelet count below $75,000 / \mathrm{mm}^{2}$ was obtained, chemotherapy was postponed, and radiotherapy was performed instead. The initial radiation therapy was performed five times a week at an irradiation dosage of 1.8-2 Gy using a linear accelerator ( $6 \mathrm{mV}$ photons) 4-5 days after completing chemotherapy. A 3D conformal shrinking field was used, and the radiation technique was provided along with the duration of the total course of treatments. A total 36 Gy was administered into the base of the skull and supraclavicular fossa. Additional radiation (20-30 Gy) was administered to a smaller area a week after the second course of chemotherapy.

Progression of the primary lesion in the nasopharynx was evaluated using CT, MRI and fibre-optic pharyngeal endoscopy. Metastasis to cervical lymph nodes was evaluated using CT, MRI and palpation. The evaluation of distant metastasis was performed using CT, liver ultrasonography, upper gastrointestinal tract endoscopy and bone scintigraphy. Since 2006, PET (positron emission tomography) CT, or PET-MRI has also been used to evaluate the extent of disease. Treatment completion, response and five-year survival rates of each ACRT-treated patient were calculated from medical records, and the results were compared to previously published data.

\section{Results}

Of the 25 patients, 24 (96\%) underwent all three courses of chemotherapy and scheduled radiation doses; thus, there was a $96 \%$ treatment completion rate in our study. One patient refused further treatment after it was determined there was a complete response (CR) after additional radiotherapy. In our study, the most common haematological side effect, at grade 3 toxicity or above, was neutropenia (three patients), and the most common non-haematological symptoms were nausea and mucositis (four patients). The toxicity profile is described in Table II, which was assessed before the third course of chemotherapy using Common Terminology Criteria for Adverse Events (CTCAE v. 4.0). Of note, there were no fatal toxicities due to ACRT, corroborating previous work ${ }^{2}$. Distant metastases were revealed in five patients after ACRT. Of the five patients, one patient died, and four patients survived due to surgery, chemotherapy, or radiation therapy interventions. Local recurrences were revealed in the nasopharynx of five patients, and of these, four patients died, with one patient surviving following laser resection. Three patients received a neck dissection for cervical lymph node metastasis. In one patient, this metastasis persisted after the conclusion of ACRT, and the patient died of disease at seven months after neck dissection. In one patient, cervical lymph node metastasis was revealed six months after therapy, and the patient died of gastric cancer 24 months later. In the other patient, cervical lymph node metastasis was revealed 14 months after therapy, with the patient surviving for over eight years after receiving surgery for lung, liver and bone metastases. There were no cases requiring reduced dosages of chemotherapy drugs. Additionally, ACRT radiation exposure dynamics did not need to be modified, and percutaneous gastrostomy tubes were not placed before or during ACRT. The total doses of radiotherapy administered to each patient and their outcomes are described in Table III. Although dosage of radiotherapy in our ACRT regimen for nasopharyngeal cancer is lower than that of other chemoradiotherapy regimens, and the only patients who was treated with lower doses of radiotherapy did not tend to experience

Table II. Adverse effects during ACRT. Common terminology criteria for adverse events (CTCAE v. 4.0).

\begin{tabular}{lcccc} 
& \multicolumn{5}{c}{ Toxicity grade (number of patients) } \\
& $\mathbf{1}$ & $\mathbf{2}$ & $\mathbf{3}$ & $\mathbf{4}$ \\
Haematologic & & & & \\
White blood cell & 8 & 14 & 3 & 0 \\
Neutrophil & 9 & 13 & 3 & 0 \\
Platelet & 24 & 1 & 0 & 0 \\
Non-haematologic & & & & \\
Nausea & 10 & 11 & 4 & 0 \\
Mucositis & 11 & 10 & 4 & 0 \\
Renal dysfunction & 23 & 2 & 0 & 0 \\
\hline
\end{tabular}

ACRT $=$ Alternating chemoradiotherapy 
Table III. Total doses of radiotherapy administered to each patient and their outcomes.

\begin{tabular}{lcccc}
$\begin{array}{l}\text { Total doses } \\
(G y)\end{array}$ & $\begin{array}{c}\text { Number } \\
\text { of patients }\end{array}$ & $\begin{array}{c}\text { Local } \\
\text { recurrence }\end{array}$ & $\begin{array}{c}\text { Neck } \\
\text { metastasis }\end{array}$ & $\begin{array}{c}\text { Clinical } \\
\text { course }\end{array}$ \\
54.4 & 2 & 0 & 0 & NED 2/2 \\
56 & 1 & 0 & 0 & NED \\
56.4 & 4 & 2 & 0 & NED 1/4 \\
& & & & DOD 2/4 \\
& 1 & 0 & 0 & NOOD 1 \\
58.2 & 1 & 0 & 0 & NED \\
58.4 & 1 & 0 & 0 & NED \\
59.6 & 2 & 0 & 0 & NED 2/2 \\
60 & 4 & 0 & 1 & NED 4/4 \\
60.4 & 1 & 1 & 0 & DOD \\
61.2 & 1 & 0 & 1 & DOOD \\
62.4 & 1 & 0 & 0 & NED \\
63 & 1 & 0 & 0 & Alive wD \\
66 & 4 & 2 & 1 & NED 3/4 \\
66.4 & 1 & 0 & 0 & DOD 1/4 \\
70 & 1 & & & NED \\
\hline
\end{tabular}

$\overline{N E D}=$ no evidence of disease; Alive $W D=$ alive with disease; $D O O D=$ died of other disease; $D O D=$ died of primary disease.

local recurrence. Thus, in sum, the five-year progressionfree survival (PFS) rate was 70\%, and the five-year overall survival rate was $89.3 \%$ (Fig. 2).

\section{Discussion}

When an initial diagnosis of nasopharyngeal cancer is made, it typically lacks specific symptoms and is often in an advanced stage. Previous studies have shown that combined chemotherapy is better than radiotherapy alone for treatment of nasopharyngeal cancer ${ }^{3-7}$. For example, PFS and overall survival (OS) rates are significantly improved with concurrent chemoradiotherapy (CCRT) plus adjuvant therapy compared with RT alone. The three-year survival rate for RT patients was $47 \%$ compared with a $78 \%$ three-year survival rate for CCRT plus adjuvant therapy $(\mathrm{P}=0.005)$, and three-year PFS was $24 \%$ versus $69 \%$, respectively $(\mathrm{P}<0.001)$. However, in these studies, the treatment period was longer (130 days), and the treatment completion rate was lower $(55 \%)$ in patients treated via CCRT combined with adjuvant therapy ${ }^{23}$. Other combinations of radiotherapy and chemotherapy have been used to treat nasopharyngeal cancer followed by an initial round of radiation. In particular, several methods are used including adjuvant chemotherapy ${ }^{89}$, neo-adjuvant chemotherapy ${ }^{1011}$, ACRT ${ }^{1712-1420}$ and CCRT ${ }^{3515-18}$. It appears that the regimen used is different in each institution, including the administration time of the individual drugs. In the present study, ACRT was performed with a treatment completion rate of $96 \%$ in our department. Previous ACRT treatment completion rates from other institutions were $80 \%, 70 \%$ and $76.1 \%{ }^{13} 1420$, and CCRT completion rates were $63 \%$ and $66 \%{ }^{3}{ }^{17}$. ACRT has been previously reported to result in fewer side effects compared to CCRT ${ }^{13}$. Therefore, we administered both a drug and radiation dose in our ACRT regimen that minimised side effects. Compared to the ACRT regimen outlined by Fuwa et al. ${ }^{13}$, additional radiation was reduced in our study if the patient had a CR after the second course of chemotherapy. At our institution, the total radiation dose ranged from 54.4 to 70 Gy (average: $60.7 \mathrm{~Gy}$ ). Although the standard irradiation dose is 66-70 Gy in most regimens ${ }^{414}, 60 \mathrm{~Gy}$ was chosen for the curative irradiation dose based on previous studies of the synergistic interaction between chemotherapy and radiotherapy ${ }^{1321}$. As a result, the lower dosage of radiotherapy might have contributed to the higher treatment completion rate, and thus to the higher survival rates. Although the dosage of radiotherapy in our ACRT regimen for nasopharyngeal cancer is lower than that of other chemoradiotherapy regimens, the treatment completion rate, response rate and $\mathrm{CR}$ rate were all high, and the survival rate might have enough power to allow comparison with other studies.

Nasopharyngeal cancer is most common in East Asia, Japan included, and EBV is mentioned among the risk factors for nasopharyngeal cancer. Although the link between EBV infection and nasopharyngeal cancer is complex and not completely understood, the success of this low dose radia-

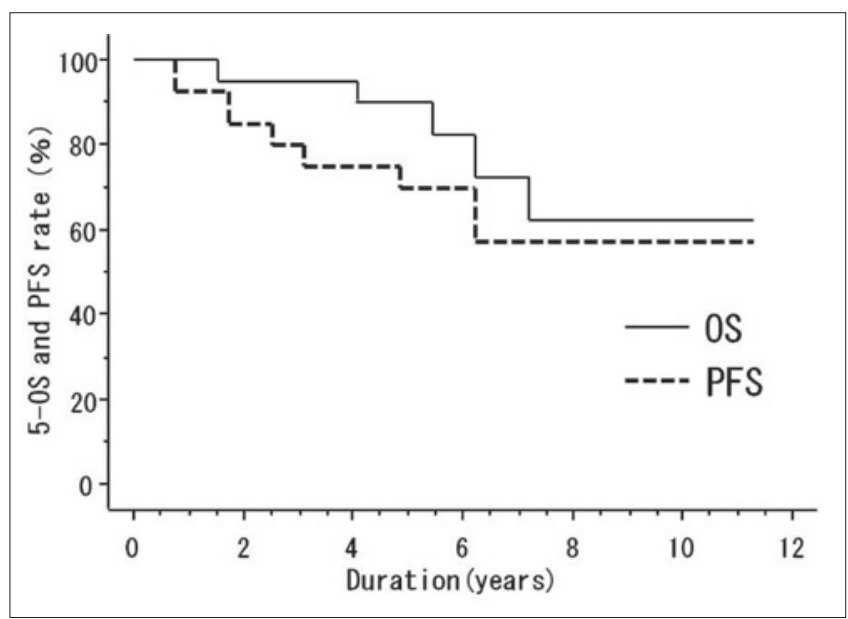

Fig. 2. 5-year overall survival (OS) and progression-free survival (PFS) of nasopharyngeal cancer patients who underwent alternating chemoradiotherapy in our institution by the Kaplan-Meier method. 5-year OS and PFS were $89.3 \%$ and $70 \%$. 
tion procedure might have been related to a factor specific to East Asian nasopharyngeal cancer. In addition, to improve ACRT treatment completion rate, the interval between radiation and chemotherapy was maintained in our study in order to control vital signs, symptoms, renal function, bone marrow activity and patient recovery. Similar to an original paper by Fuwa et al. ${ }^{13}$, when serum creatinine levels were above $1.5 \mathrm{mg} / \mathrm{dL}$ on the scheduled date of chemotherapy, chemotherapy was not performed. In addition, when a WBC count below $3,000 / \mathrm{mm}^{2}$ or platelet count below $75,000 / \mathrm{mm}^{2}$ was obtained, chemotherapy was postponed, and radiotherapy was performed instead. If necessary, at our institution, we waited three weeks to meet the inclusion criteria (WBC count $>3,000 / \mathrm{mm}^{2}$ and platelet count $>75,000 / \mathrm{mm}^{2}$ ) after the initial round of chemotherapy. We used G-CSF and consulted the internal medicine department to determine the next steps regarding the patient's administration of subsequent rounds of chemotherapy. Because it is important that all patients complete the third systemic course of chemotherapy, patients who refused this third course had a poor prognosis, as previously noted ${ }^{14}$.

In addition, when the WBC and platelet counts were respectively below $1,000 / \mathrm{mm}^{2}$ and $25,000 / \mathrm{mm}^{2}$ after chemotherapy, the dose of 5-FU and CDDP was decreased by $25 \%$ at the next administration of chemotherapy. Moreover, when serum creatinine levels temporarily exceeded $1.5 \mathrm{mg} / \mathrm{dL}, 5-\mathrm{FU}$ and CDDP were decreased by $25 \%$.

As mentioned, the CR rate in our study was $96 \%$. A previous study highlighted an ACRT CR rate of $84 \%{ }^{13}$, and other studies noted CCRT CR rates of $70 \%$ and $93 \%{ }^{16} 18$. With respect to the antitumour effects, ACRT is inferior to CCRT; however, in general, the CR rate is higher. This highlights the fact that completion of ACRT is extremely important. Moreover, the five-year survival rate was $89.3 \%$ in our study. Two previous studies showed that the three-year survival rate at institutions that performed neo-adjuvant chemotherapy was $58 \%$ and $59 \%$, respectively ${ }^{10}{ }^{11}$. Additional studies highlighted the following survival rates with respective therapies: adjuvant chemotherapy, 59\% (four-year survival rate) ${ }^{8}$ and $61 \%$ (fiveyear survival rate) ${ }^{9}$; CCRT, $72 \%$ and $70.3 \%$ (five-year survival rates) ${ }^{519}$; and ACRT, $78.1 \%, 83 \%$, and $78.04 \%$ (five-year survival rates) ${ }^{12} 1320$.

It has also been reported that a lower CDDP dose is more effective. This is because the treatment completion rate and survival rate over three years using a lower CDDP dose were both higher compared with treatment completion and survival rates using higher CDDP doses ${ }^{13}$. The extent to which the CDDP dosage can be reduced is unknown; however, ACRT demonstrates decreased invasiveness as compared with CCRT ${ }^{13}$. Moreover, for nasopharyngeal cancer, a wider irradiation field is required during radiotherapy because of its progression pattern, and thus, mucositis is a concerning problem when radiotherapy alone is performed. Considering these characteristics of nasopharyngeal cancer, ACRT may be a useful method for treating nasopharyngeal cancer because a sufficient number of antitumour agents can be administered in combination with radiotherapy. Thus, mucositis can be decreased in the ACRT regimen as compared with CCRT ${ }^{15}$.

Prior to ACRT, we explained to patients the potential benefits in an attempt to increase willingness towards treatment and encourage full completion. There are several reports that a majority of individuals are not diagnosed with nasopharyngeal cancer until they are in stage IV ${ }^{13}$, and $40 \%$ of the cases in our department were also detected at stage IV. This is because nasopharyngeal cancer does not typically present with specific symptoms as stated earlier. However, it can be rapidly diagnosed in departments such as ours if nasal congestion and the ears are affected compared with presentation of symptoms such as headache and eye problems. Additionally, nasopharyngeal cancer is a rare form of head and neck cancer and is therefore not included as a differential diagnosis for many nonspecific symptoms. To address the issues of delayed diagnosis in the future, physicians should consider this type of cancer when patients present with persisting facial symptoms.

It is also important that a multidisciplinary approach (i.e., ACRT) is continuously employed in the treatment of distant metastasis after a neck dissection has been done to control such metastasis. Furthermore, the primary tumour recurrence rate is associated with poor prognosis, and thus, the choice of treatment is restricted due to anatomical reasons ${ }^{12}$. Split course radiation has inferior treatment outcomes. However, ACRT is different from split course radiation therapy because of the subsequent course of chemotherapy. In addition, ACRT permits patients to take higher doses of antitumour agents compared with CCRT ${ }^{14}$.

\section{Conclusions}

In conclusion, we demonstrate that ACRT is an effective regimen to treat nasopharyngeal cancer, revealing higher treatment completion, response and five-year overall survival rates compared with other combinatorial radiotherapy and chemotherapy treatment regimens. Because our results are based on a clinical trial with only 25 patients, further study will be required to draw firm conclusions. 


\section{References}

1 Fuwa N, Ito Y, Kodaira T, et al. Therapeutic results of alternating chemoradiotherapy for nasopharyngeal cancer using cisplatin and 5-fluorouracil: its usefulness and controversial points. Jpn J Clin Oncol 2001;31:589-95.

2 Rosenthal DI. Consequences of mucositis-induced treatment breaks and dose reductions on head and neck cancer treatment outcomes. J Support Oncol 2007;5:23-31.

3 Al-Sarraf M, LeBlanc M, Giri PG, et al. Chemoradiotherapy versus radiotherapy in patients with advanced nasopharyngeal cancer: phase III randomized Intergroup study 0099. J Clin Oncol 1998;16:1310-7.

4 NCCN Guidelines Version 2. 2014 Cancer of the Nasopharynx. NCCN.org

5 Lin JC, Jan JS, Hsu CY, et al. Phase III study of concurrent chemoradiotherapy versus radiotherapy alone for advanced nasopharyngeal cancer: positive effect on overall and progression-free survival. J Clin Oncol 2003;21:631-7.

6 Marcial VA, Hanley JA, Chang C, et al. Split-course radiation therapy of carcinoma of the nasopharynx: results of a national collaborative clinical trial of the Radiation Therapy Oncology Group. Int J Radiat Oncol Biol Phys 1980;6:409-14.

7 Kawashima M, Fuwa N, Myojin M, et al. A multi-institutional survey of the effectiveness of chemotherapy combined with radiotherapy for patients with nasopharyngeal carcinoma. Jpn J Clin Oncol 2004;34:569-83.

8 Rossi A, Molinari R, Boracchi P, et al. Adjuvant chemotherapy with vincristine, cyclophosphamide, and doxorubicin after radiotherapy in local-regional nasopharyngeal cancer: results of a 4-year multicenter randomized study. J Clin Oncol 1988;6:1401-10.

9 Chi KH, Chang YC, Guo WY, et al. A phase III study of adjuvant chemotherapy in advanced nasopharyngeal carcinoma patients. Int J Radiat Oncol Biol Phys 2002;52:1238-44.

10 Chua DT, Sham JS, Choy D, et al. Preliminary report of the asian-oceanian clinical oncology association randomized trial comparing cisplatin and epirubicin followed by radiotherapy alone in the treatment of patients with locoregionally advanced nasopharyngeal carcinoma. Asian-Oceanian Clinical Oncology Association Nasopharynx Cancer Study Group. Cancer 1998;83:2270-83.
11 Chan AT, Teo PM, Leung TW, et al. A prospective randomized study of chemotherapy adjunctive to definitive radiotherapy in advanced nasopharyngeal carcinoma. Int J Radiat Oncol Biol Phys 1995;33:569-77.

12 Goto Y, Kodaira T, Fuwa N, et al. Alternating chemoradiotherapy in patients with nasopharyngeal cancer: prognostic factors and proposal for individualization of therapy. J Radiat Res 2013;54:98-107.

13 Fuwa N, Shikama N, Hayashi N, et al. Treatment results of alternating chemoradiotherapy for nasopharyngeal cancer using cisplatin and 5-fluorouracil - a phase II study. Oral Oncol 2007;43:948-55.

14 Ueno T, Endo K, Kondo S, et al. Factors affecting outcomes of alternating chemoradiotherapy for nasopharyngeal cancer. Ann Otol Rhinol Laryngol 2014;123:509-16.

15 Lee AW, Lin JC, Ng WT. Current management of nasopharyngeal cancer. Semin Radiat Oncol 2012;22:233-44.

16 Bae WK, Hwang JE, Shim HJ, et al. Phase II study of docetaxel, cisplatin, and 5-FU induction chemotherapy followed by chemoradiotherapy in locoregionally advanced nasopharyngeal cancer. Cancer Chemother Pharmacol 2010;65:589-95.

17 Lee AW, Tung SY, Chua DT, et al. Randomized trial of radiotherapy plus concurrent-adjuvant chemotherapy vs radiotherapy alone for regionally advanced nasopharyngeal carcinoma. J Natl Cancer Inst 2010;102:1188-98.

18 Xiao WW, Huang SM, Han F, et al. Local control, surviv$a l$, and late toxicities of locally advanced nasopharyngeal carcinoma treated by simultaneous modulated accelerated radiotherapy combined with cisplatin concurrent chemotherapy.Long-term results of a phase II study. Cancer 2001;117:1874-83.

19 Chan AT, Leung SF, Nqan RK, et al. Overall survival after concurrent cisplatin-radiotherapy compared with radiotherapy alone in locoregionally advanced nasopharyngeal carcinoma. J Natl Cancer Inst 2005;97:536-9.

20 Fuwa N, Kodaira T, Daimon T, et al. The long-term outcomes of alternating chemoradiotherapy for locoregionally advanced nasopharyngeal carcinoma: a multiinstitutional phase II study. Cancer Med 2015;4:1186-95.

21 Fujii M, Tsukuda M, Satake B, et al. Phase I/II trial of weekly docetaxel and concomitant radiotherapy for squamous cell carcinoma of the head and neck. Int J Clin Oncol 2004;9:107-12.

Received: May 31, 2017 - Accepted: September 1, 2017

Address for correspondence: Satoshi Saijoh, Fukushima Medical University, School of Medicine, Department of Otolaryngology, 1 Hikarigaoka, Fukushima City 960-1295, Japan. Tel. +81 245471325. Fax +81 245483011. E-mail: satoshi.saijoh@gmail.com 